\title{
Propuesta de tipología básica de los videojuegos de PC y consola
}

\author{
Rafael Carrasco Polaino
}

Profesor de Tecnología de la Información, Empresa Informativa e Informática Aplicada a la Comunicación. Centro Universitario Villanueva, adscrito a la Universidad Complutense de Madrid y ESIC.

\section{Resumen}

Identificar de forma sencilla los principales tipos de videojuegos tanto en soporte PC como videoconsola es importante para poder, a partir de ahí, estudiar cómo se acercan los usuarios a este sector de entretenimiento y cómo es su relación con cada uno de los tipos de juegos. Sólo de esta forma se podrá conocer cuáles son las consecuencias positivas o negativas que pueden ejercer esta actividad sobre los usuarios.

\section{Palabras clave}

Videojuego - Videoconsola - Ordenador - Entretenimiento - Ocio - Juventud

\section{Abstract}

To identify easily the main types of videogames as much in support PC as videoconsole is important to study how the users approach to this sector of entertainment and how it is their relation with each one type of games. Only there it will be possible to know positive or negative consequences that can exert this activity on the users. 


\section{Key words}

Videogame - Videoconsole - Personal computer - Entertainment - Leisure - Youth

\section{Introducción}

Las posibles consecuencias positivas o negativas que tienen los videojuegos sobre los jóvenes y adolescentes es un tema que durante los últimos años ha tenido repercusiones en gran numero de estudios y en medios de comunicación. El medio argentino La Capital indica, por ejemplo, que los videojuegos pueden volver solitarios y enfermos a los niños ${ }^{1}$. También se puede comprobar esta preocupación en el medio Granada Hoy cuando en una de sus informaciones publica que "Casi la mitad de los niños de 10 años ya tiene problemas con el sobrepeso ${ }^{2 \text { ” }}$ explicando que una de las posibles consecuencias de esto sea la gran cantidad de horas que estos niños pasan delante del monitor o de la televisión, jugando. Incluso se pueden ver argumentos tan peligrosos como los que sostiene Amnistía Internacional, cuando sostiene que los padres no son capaces de controlar a sus hijos en relación a este aspecto ${ }^{3}$.

Por otro lado, José Cervera, autor del conocido blog Retiario afirma que "El 100\% de los

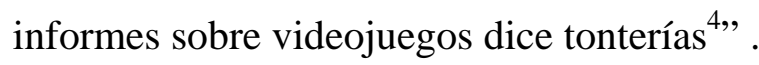

Sin embargo, no se pueden sostener afirmaciones de carácter general, ya que no todos los videojuegos son iguales. Los hay de infinidad de tipos y modelos, más violentos, más rápidos, más agresivos, pero también más tranquilos, más estratégicos y más reflexivos. Los hay para jugar solo, pero también para jugar en compañía o para jugar en equipo, y cada uno de estos tipos de juegos deben ofrecer unas consecuencias diferentes, ya sean positivas o negativas, y esto es lo que se va a hacer a los largo de este texto, identificar los diferentes tipos de videojuegos.

Antes de comenzar a describir los diferentes tipos de videojuegos que se pueden encontrar en el mercado tanto en soporte ordenador personal como en consola, debemos indicar que esta clasificación se desarrolla atendiendo a la variable “técnica de juego” antes que al concepto temática.

\footnotetext{
${ }^{1}$ Ref. http://www.lacapital.com.ar/2006/06/11/general/noticia_301026.shtml

${ }^{2}$ Ref. http://www.diariogranadahoy.com/diariogranadahoy/articulo.asp?idart=2948300\&idcat=2911

${ }^{3}$ Ref.

http://www.es.amnesty.org/cms/index.php?\&id=12\&no_cache=1\&tx_ttnews\%5btt_news\%5d=606\&tx_ttnews\%5bbackP id $\% 5 d=61$

${ }^{4}$ Ref. http://blogs.20minutos.es/retiario/post/2006/01/17/el-100-los-informes-sobre-videojuegos-dice-tonterias
} 
El Profesor Pere Marqués Graells de la Universidad Autónoma de Barcelona establece una clasificación bastante clara a partir de la cual podremos establecer la nuestra con más detalle. El profesor identifica seis tipos de videojuegos:

- Arcades

- Aventura y Rol

- Simuladores

- Estrategia

- Deportes

- Puzzles y preguntas

Comencemos a describir y completar nuestra propia clasificación:

\section{Tipología de videojuegos}

\subsection{Arcades}

Un videojuego de este tipo se define como juego de animación que requiere mucha rapidez de reacción. EL personaje animado supera pantallas de creciente dificultad. Aquí planteamos una subdivisión en dos tipos de arcades diferentes: los juegos de plataformas y los juegos de acción. Estos últimos también los podemos subdividir en Fist Person Shooter (FPS) y juegos de acción en tercera persona.

- Plataformas

- Acción

o First Person Shooter (FPS)

o Acción en tercera persona

En los juegos de plataformas el personaje debe ir avanzando a lo largo de las diferentes pantallas salvando obstáculos, saltando y eliminado o esquivando a aquellos enemigos que impiden el progreso. El juego, según se avanza, va ganando en dificultad, que suele reflejarse en un aumento de la velocidad, por lo que el jugador debe cada vez reaccionar de forma más rápida cada vez.

Son juegos de mucho éxito entre los más jóvenes y público femenino, aunque dependiendo de la temática, abarca segmentos de usuarios más amplios. Destacan Mario Bros., por ser uno de los primeros con multitud de secuelas y que todavía tiene gran número de seguidores, Prince of Persia, como un clásico del que acaba de aparecer una primera secuela y del que se está preparando una 
segunda o la saga Tomb Raider, que aunque teniendo un alto componente de plataformas, lo incluiremos entre los juegos de acción en tercera persona.

Los FPS son videojuegos de acción en primera persona en donde el usuario se integra con el personaje del juego. Lo que aparece en la pantalla o televisor es la mano del personaje portando el arma que lleve en ese momento. Estos juegos son de gran variedad temática y se suelen desarrollar sobre un guión o historia cada vez más cinematográfica. La base del desarrollo del juego es la supervivencia a lo largo de los diferentes niveles matando a cualquier enemigo que se interponga en el camino. Una de las características que determinan la calidad del videojuego es la interacción con los escenarios. Cuantos más elementos del escenario pueda coger o destruir el personaje, mejor y más completo se considera el juego.

Los juegos de más éxito de este tipo pueden ser la saga Doom, uno de los primeros FPS de la historia, aunque su tercera parte es del año 2005, Half Life o Halo. Son juegos con un alto contenido de violencia y sangre.

Los juegos de acción en tercera persona se diferencian de los anteriores en la forma de integración entre el jugador y el personaje. En este caso el personaje no se identifica tanto con el jugador. Se ve al personaje de forma completa en la pantalla. Es ajeno al usuario. Juegos en los que la acción se ve relegada por la estrategia, el sigilo. Resultan algo más lentos de jugar y la historia o guión está más elaborada. Contienen un alto componente de plataforma.

De entre estos videojuegos podemos destacar la saga de Tomb Raider o la de Metal Gear Solid.

\subsection{Aventura y Rol}

Aunque el profesor Marqués los unifica dentro de la misma categoría, son juegos con grandes diferencias tanto en jugabilidad como temática como en la forma de relacionarse el jugador con el videojuego.

En los juegos de aventura gráfica predominan los diálogos y la interacción con los elementos del escenario o con otros personajes. Son juegos lentos, en los que se necesita paciencia e introducirse mucho en el desarrollo para poder solucionar los diferentes enigmas y puzzles que se van proponiendo y así conseguir que la historia se siga desarrollando y el jugador pueda avanzar. Son muy absorbentes y favorecen el diálogo con otros jugadores para explicar cómo se ha conseguido superar algún enigma. 
Dentro de este tipo de juegos destacan las sagas de Monkey Island o Broken Sword.

En los juegos de Rol o RPG (Rol Player Game) se debe actuar y tomar decisiones en función de las propuestas del juego y de las acciones del resto de personajes. Se mantiene en todo momento un control exhaustivo de las constantes vitales, habilidades, armas y poderes y demás características del personaje. Son juegos de temática mágica, y anacrónica.

Su diseño propone una jugabilidad lenta. Se diferencia de los juegos de acción en que el desarrollo de las luchas es por turnos y el jugador dedica más tiempo a planificar la estrategia del ataque que en la acción en si.

Dentro de este tipo de juego destaca la saga Final Fantasy. Juego con más de diez secuelas y que ha tenido su repercusión en el cine con la aparición de una película de animación por ordenador que lleva el mismo título.

\subsection{Simuladores}

Son juegos de gran veracidad y realismo para entornos y situaciones reales. El usuario debe aplicar en ocasiones conocimientos específicos.

En estos videojuegos el usuario se introduce en algún tipo de transporte, ya sea coche, motocicleta o avión y debe pilotarlo completando algún recorrido, sorteando diferentes obstáculos o ganando carreras 5 .

El grado de integración del jugador en el entorno es variable, ya que la visión puede ser en tercera o en primera persona.

La temática de más éxito es la de las carreras de coches. Juegos en donde lo que importa en vencer en competiciones que se corren por circuitos, carreteras o calles urbanas sin importar las normas de circulación vial. Esto podría resultar absurdo tratándose de una carrera, pero no lo es tanto cuando observamos con detalle y comprobamos los recursos que han destinado los desarrolladores en detallar las señales de tráfico, los semáforos o las marcas viales.

\footnotetext{
5 ¿Quién quiere ser maquinista?

Fecha: 23/08/2005

Friendware lanza un nuevo videojuego, 'EEP Virtual Railroad 3' para ordenador en el que ofrece la oportunidad a los jugadores de convertirse en maquinistas ferroviarios. Aquellos usuarios que tenga pasión por las viejas locomotoras del pasado y los modernos trenes de alta velocidad, ya tienen su gran oportunidad, con la que planificarán, construirán, viajarán y controlarán todo el sistema ferroviario.

http://www.elmundo.es/navegante/2005/08/23/juegos/1124790641.html
} 
Son también juegos con algún contenido sexual ${ }^{6}$, no tanto a la hora de jugar como en su promoción en y los videos presentación, donde aparecen mujeres con poca ropa muy ajustada y proporciones irreales.

Aquellos de más éxito son los que más detalles cuidan a la hora de programar los coches. Son modelos reales a los que el jugador mientras progresa en el juego, puede ir acoplando complementos para aumentar la potencia y la velocidad y así seguir progresando.

Los juegos de más éxito de este tipo son Need for Speed y Grand Turismo o Grand theft Auto.

\subsection{Estrategia}

En este tipo de juegos se promueve la toma de decisiones, cálculos, razonamiento lógico, establecimiento de hipótesis, etc. para conseguir un objetivo.

Suelen ser de tema histórico y épico donde se recrean las principales civilizaciones de la historia, El jugador debe, a partir de unos pocos elementos de construcción y personajes, ir construyendo una ciudad con todos sus componentes para crear sus ejércitos y poder enfrentarlos a los ejércitos de otro jugador, vencer y marchar a destruir la ciudad del oponente.

Aunque la temática más importante es la de carácter histórico, también destaca aquella relacionada con el espacio exterior y extraterrestres en donde se enfrentan diferentes razas o de carácter mágico y anacrónico.

Existen dos tipos claramente diferenciados de juegos de estrategia: en tiempo real y por turnos.

En los juegos en tiempo real todos los jugadores van realizando sus acciones a la vez, mientras que en los juegos por turnos los jugadores van realizando sus diferentes movimientos uno detrás de otro. Esta es una característica muy importante a la hora de analizar como se relaciona el jugador con el videojuego ${ }^{7}$.

6 'GTA San Andreas', sólo para adultos por su 'contenido sexual explícito' oculto Fecha: 22/07/2005

El videojuego 'Grand Theft Auto: San Andreas', en el ojo del huracán por las las numerosas críticas, entre ellas las de la senadora estadounidense Hillary Clinton, a causa de su contenido sexual oculto, ha sido recalificado como "sólo para adultos". Esto demuestra, además, que la modificación 'Hot Coffee' que circulaba por la Red desbloqueaba un 'contenido sexual explícito' que ya existía en el juego, en su versión para PC. http://www.elmundo.es/navegante/2005/07/22/juegos/1122020504.html 7 Lucha sin tregua en la Grecia clásica Fecha: 07/06/2005 
Cuando hablamos de tiempo real, importa tanto el tipo de movimiento como la velocidad con la que lo hacemos, siempre antes que el oponente para avanzar más deprisa, terminar antes de construir la ciudad y los ejércitos y poder lanzarlos contra el adversario antes de que termine sus herramientas de juego. Debido a esta característica, el aspecto de la calidad gráfica no adquiere tanta importancia.

Los videojuegos de estrategia por turnos sin embargo, son muy diferentes en este aspecto. El jugador dedica más tiempo a la elaboración de la jugada que al desarrollo de la acción de la misma. El usuario sólo participa en la elaboración y no en la acción, la acción la realiza la máquina a través de un video que recrea una simulación de la estrategia y sus efectos. Por esto el juego es mucho más lento, la participación del usuario más pasiva y cobra mayor importancia el apartado gráfico. Podríamos decir que es similar a un juego de mesa pero con gran despliegue de efectos a través de la pantalla.

Existen multitud de títulos de juegos de estrategia en tiempo real. Podemos destacar sagas como Age of Empires, Patrician, Imperium ${ }^{8}$, Warcraft, Starcraft...

Los juegos de estrategia por turnos aparecieron antes que los de tiempo real y han mutado hacia los RPG, pero dos de estos juegos siguen teniendo gran auge y generando comunidades de usuarios, estos son Civilitation y Populous.

Según Brian Reynolds 'El jugador tiene que sentir que está entrando en un mundo de fantasía' ${ }^{\prime 9}$.

\footnotetext{
La mitología griega se ha convertido en uno de los escenarios más excitantes para los desarrolladores de videojuegos. Es el caso de 'God of War', la nueva creación de Sony Computer Entertainment España, videojuego en el que el reino humano y el mundo de los dioses se entremezclan en una atmósfera en la que priman la fuerza bruta y la violencia. Pero no sólo existe acción, ya que los jugadores también deberán hacer uso de su destreza mental y su capacidad para descifrar enigmas.

http://www.elmundo.es/navegante/2005/06/07/juegos/1118156570.html

8 'Imperium III' crea 'entusiastas de la Historia'

Fecha: 16/12/2004

La decana de la facultad de Geografía e Historia de la Universidad Complutense de Madrid, Mercedes Molina, aseguró que el videojuego 'Imperium III. Las grandes batallas de Roma es una gran herramienta divulgativa' y crea 'entusiastas de la Historia'.

http://www.elmundo.es/navegante/2004/12/16/juegos/1103212223.html

9 Brian Reynolds, diseñador y programador, es una mente privilegiada para el desarrollo de videojuegos que ha pasado por compañías como Microprose o Firaxis y que en la actualidad trabaja con Douglas Kaufman en Big Huge, su nueva aventura empresarial.. Ref. elmundo.es
} 


\subsection{Deportes}

Los juegos de deportes siempre han tendido un gran éxito, sobre todo los relacionados con el fútbol. Estos videojuegos han evolucionado de basarse en el enfrentamiento entre dos equipos donde un partido decide la partida, hasta los juegos actuales en donde el usuario puede gestionar todo un equipo como si fuera un presidente de un club deportivo decidiendo inversiones, fichajes, traspasos y cesiones, etc. incluso algunos de estos juegos han llegado a limitar la posibilidad de que el jugador participe en los partidos, dejando al ordenador y a la consola que calcule el resultado de un partido en función de cómo lo ha planteado tácticamente el usuario.

De esta forma los juegos de deportes se han visto desdoblados en su jugabilidad. Un tipo se basa en la gestión y organización de un club deportivo siendo más tranquilo y lento de jugar, y otros se ha dirigido hacia la simulación de partidos en donde el jugador participa activamente en los encuentros, siendo estos juegos donde se precisa más velocidad de reflejos y de manos al manejar el teclado o el pad (mando de la consola).

Debemos destacar que todos los juegos de deportes son casi idénticos, en sus programación y motor gráfico. La jugabilidad es la misma. Sólo cambian los escenarios y el modelado de los personajes.

Otro tipo de juegos basado en los deportes son los de atletismo, los destacamos porque en este tipo de videojuegos lo que importa es la velocidad con la que pulsemos alternativamente dos teclas o dos botones del pad. Cuanto más rápidamente pulsemos las teclas, más correrá el personaje. No importa la planificación ni la técnica, tan sólo la velocidad de dedos.

Algunos títulos de gran éxito son PC Futbol, Pro Evolution Soccer o FIFA Futbol.

\subsection{Puzzles y preguntas}

Estos juegos fueron los primeros en ser creados y los que durante más tiempo estuvieron en auge porque las necesidades graficas que debía tener el usuario para jugar no son demasiado altas y porque el merado de los videojuegos no tenía aún el potencial que tiene en la actualidad.

Son videojuegos que han pasado de moda y a los que ya no se juega. Han quedado fuera de los hogares para terminar en una esquina de los salones recreativos o de algún pub. Sin embargo suelen surgir algunos títulos que imitan la jugabilidad y técnica de aquellos clásicos y que adquieran un gran éxito durante un corto periodo de tiempo. 
Sin embargo títulos como Tetris o Comecocos sirvieron para que los empresarios de este sector se dieran cuenta del potencial empresarial que tenía el sector de los videojuegos y decidieran invertir en él, hasta llegar a los títulos que conocemos hoy.

\section{Consideraciones generales}

A continuación se exponen algunas ideas de carácter general que quizás ayuden a entender mejor el sector de los videojuegos, tanto de ordenador como de consola.

Los juegos más novedosos siempre son los más vendidos. Cada juego de éxito que sale a la luz bate récords de ventas. Esto indica el cada vez mayor número de usuarios que hay en el sector de los videojuegos.

\begin{tabular}{|l|l|l|l|l|}
\hline \multicolumn{5}{|c|}{ Juegos más vendidos durante el 2005 (ordenados por ventas) } \\
\hline Plataforma & \multicolumn{1}{|c|}{ Título } & \multicolumn{1}{|c|}{ Distribuidor } & \multicolumn{1}{c|}{ Genero } & Edad \\
\hline PS2 & PRO EVOLUTION SOCCER 5 & KONAMI & SPORT & $3+$ \\
\hline GBA & POKEMON ESMERALDA & NINTENDO & GRAPH.ADV./RPG & $3+$ \\
\hline PS2 & GRAN TURISMO 4 & SONY & RACE/RALLY & $3+$ \\
\hline PS2 & GRAND THEFT AUTO: SAN ANDREAS & TAKE TWO & ACTION/COMBAT & $18+$ \\
\hline PS2 & PRO EVOLUTION SOCCER 4 & KONAMI & SPORT & $3+$ \\
\hline PS2 & FIFA 06 & ELECTRONIC ARTS & SPORT & $3+$ \\
\hline PC & LOS SIMS 2 & ELECTRONIC ARTS & STRATEGY & $7+$ \\
\hline PS2 & NEED FOR SPEED: MOST WANTED & ELECTRONIC ARTS & RACE/RALLY & $3+$ \\
\hline PS2 & FORMULA ONE 2005 & SONY & RACE/RALLY & $3+$ \\
\hline PS2 & NEED FOR SPEED: UNDERGROUND 2 & ELECTRONIC ARTS & RACE/RALLY & $3+$ \\
\hline
\end{tabular}

Fuente: ADESE

Los juegos de más éxito son aquellos que generan un sentimiento de comunidad en torno a los usuarios y que desarrollan la modalidad de juego online. Aquí adquiere gran importancia el tema de los mods, modificaciones de los escenarios y personajes de los juegos basándose en el mismo motor gráfico y en la misma programación. Un ejemplo puede ser un juego de acción en primera persona ambientado en la Segunda Guerra Mundial al que se le puede aplicar un mod que transforme el juego y simule una ambientación espacial.

Es de destacar el éxito que tienen las secuelas de un juego de éxito ${ }^{10}$, secuelas que consiguen superar las ventas de la primera versión. El éxito viene determinado por el fulgurante desarrollo del mundo de la tecnología entre una versión y la siguiente.

10 Ver gráfico pag. 5. 
También resulta interesante apuntar que no todos los juegos novedosos que salen al mercado son nuevos. Es bastante común utilizar un motor gráfico de éxito para desarrollar un videojuego nuevo. Sirve como analogía utilizar el mismo motor y chasis de un coche e irle cambiando la carrocería para estrenar coche todas las semanas.

Los creadores de videojuegos dirigen sus esfuerzos hacia la calidad gráfica, intentando diseñar sus creaciones cada vez con formas más redondeadas, dirigiéndose desde polígono hacia la esfera. El problema es que un ordenador o una consola que debe mostrar el movimiento de estas formas sufre más y consume más recursos mostrando las infinitas caras de una esfera en movimiento que mostrando las seis caras de un cubo.

Las claves del éxito de los actuales y futuros videojuegos se dirigen hacia el desarrollo gráfico de los entornos y los escenarios, el desarrollo de la inteligencia artificial de los enemigos ${ }^{11} \mathrm{y}$ el desarrollo de los modos multijugador a través de Internet o de conexión local.

Por último, indicar que las empresas de videojuegos tienen, junto con los fabricantes de consolas, una estrategia comercial muy interesante que consiste en vender secuelas de éxito en exclusiva para una sola marca de consola y así conseguir vender más unidades de soporte. Un ejemplo cercano es la salida del juego Halo 2 sólo para la consola de Microsoft, $\mathrm{Xbox}^{12}$.

\section{Conclusiones}

Después de identificar los principales tipos de videojuegos y de describir algunas nociones sobre las estrategias que las empresas desarrolladoras utilizan para acercarse a los usuarios potenciales y de acto, se puede concluir que no se puede generalizar sobre las consecuencias de los videojuegos, ni positivas ni negativas. Hay cierto tipos de juegos que por su violencia y agresividad o porque premian la vulneración de las normas y leyes no son los más recomendables para aquellos

11 'La inteligencia artificial es clave en los videojuegos de próxima generación'

Fecha: 29/06/2005

La próxima generación de juegos para consolas constituirán un reto para los desarrolladores. Adam Russell, jefe de programación e Inteligencia Artificial del videojuego Fable para la consola Xbox de Microsoft, destacó durante su ponencia en el encuentro Mundos Digitales 2005 que los videojuegos del futuro exigen, cada vez más, mayores dosis de inteligencia artificial, y puso como ejemplo el archiconocido 'The Sims'. http://www.elmundo.es/navegante/2005/06/29/juegos/1120033866.html

12 "Halo 2" será uno de los platos fuertes de Windows Vista

Fecha: 10/02/2006

Microsoft quiere que su próximo sistema operativo, Windows Vista, sea un éxito. Una de las bazas de la compañía para conseguirlo son los juegos. Por ello ha anunciado que 'Halo 2', uno de los videojuegos de más éxito de la compañía, sólo estará disponible para PC bajo el nuevo sistema operativo. Además, será desarrollado por un equipo de Microsoft Game Studios en asociación con Bungie Studios.

http://www.elmundo.es/navegante/2006/02/10/empresas/1139567515.html 
más jóvenes que están en pleno proceso de socialización, pero también es cierto que hay juegos que premian la paciencia, la estrategia, la inteligencia o la participación colaborativa, y estos, dentro de un orden y de unas limitaciones temporales, puede si no ser positivo, no ser contraproducente para un crecimiento sano a nivel físico y psicológico.

Como en gran cantidad de asuntos que pueden en algunos momento causar convulsiones en la sociedad, el tema de los videojuegos como recurso de ocio en la infancia y adolescencia ha sido muy comentado en medios de comunicación y estudios, pero no se puede generalizar en cuanto a los resultados. La respuesta más correcta puede ser que un uso controlado de los videojuegos más recomendables y siempre bajo la regulación por edades de la Asociación Española de Distribuidores y Editores de Software de Entretenimiento (ADESE) ${ }^{13}$ y bajo la supervisión de los padres y educadores no tiene porque se negativo, incluso puede tener consecuencias positivas en el desarrollo psicológico de los jóvenes.

\section{Bibliografía}

- Amnistía Internacional. http://www.es.amnesty.org

- Asociación Española de Distribuidores y Editores de Software de Entretenimiento (ADESE). http://www.adese.es

- CERVERA, José. Retiario. 20 Minutos. http://www.20minutos.es

- Diario Granada Hoy. http://www.diariogranadahoy.com

- $\quad$ El Mundo. http://www.elmundo.es

- La Capital. http://www.lacapital.com.ar

\footnotetext{
${ }^{13}$ Ref. www.adese.es
} 